\title{
Avaliação multivariada da composição fenólica de cachaças envelhecidas em diferentes barris de madeira
}

\section{Multivariate evaluation of the phenolic composition of cachaça aged in barrels made of different kinds of wood}

\author{
Lidiany Mendonça ZACARONI ${ }^{1 ; 2}$; Maria das Graças CARDOSO ${ }^{3}$; Wilder Douglas SANTIAGO ${ }^{4}$; \\ João Guilherme Pereira MENDONÇA ${ }^{5}$; Cleiton Antônio NUNES ${ }^{6}$; Felipe Cimino DUARTE ${ }^{7}$ \\ ${ }^{1}$ Pesquisa financiada pelo Conselho Nacional de Desenvolvimento Científico e Tecnológico - CNPq, CAPES \\ Coordenação de Aperfeiçoamento de Pessoal de Nível Superior - CAPES e Fundação de Amparo à Pesquisa \\ do estado de Minas Gerais - FAPEMIG \\ 2 Doutoranda; Universidade Federal de Lavras - UFLA, zlidiany@yahoo.com.br \\ ${ }^{3}$ Autor para correspondência; Maria das Graças Cardoso; Doutora e Professora; Universidade Federal de Lavras \\ UFLA; Departamento de Química - DQI, Laboratório de Análises Físico-químicas de Aguardentes de Cana; \\ Campus Universitário, Caixa Postal 3037, Lavras - MG, Cep. 37.200-000, Brasil; Telefone: (35)38291202; \\ mcardoso@dqi.ufla.br \\ ${ }^{4}$ Mestrando; Universidade Federal de Lavras - UFLA, wildaoquimica@msn.com \\ ${ }^{5}$ Mestrando; Universidade Federal de Lavras - UFLA, joaoquimica@live.com \\ ${ }^{6}$ Doutor e Professor; Universidade Federal de Lavras - UFLA, cleitonnunes@dca.ufla.br \\ ${ }^{7}$ Doutorando; Universidade Federal de Lavras - UFLA, filipao_eng_alm@yahoo.com.br
}

Recebido em: 07-08-2012; Aceito em: 03-03-2014

\section{Resumo}

Este estudo objetivou avaliar a composição fenólica de cachaças envelhecidas em barris construídos de carvalho, jequitibá, castanheira, amburana, bálsamo, jatobá e louro-canela, empregando Análise de Componentes Principais (ACP). Os teores de compostos fenólicos foram centrados na média e submetidos à ACP. Os resultados indicaram que a cachaça envelhecida em castanheira (36 meses), carvalho (48 meses) e louro-canela diferiram das demais por possuirem um elevado teor de ácido gálico, ácido elágico e eugenol, respectivamente. As amostras envelhecidas em amburana (48 meses), bálsamo (36 e 48 meses), jequitibá, carvalho (24 e 36 meses) e jatobá apresentaram características semelhantes entre si, destacando a importância da análise ACP para mostrar o efeito do tipo de madeira sobre a composição fenólica de cachaça envelhecida.

Palavras-chave adicionais: cachaça; fenóis; madeira.

\begin{abstract}
This study sought to evaluate the phenolic composition of cachaças aged in barrels constructed of oak, jequitibá, chestnut, amburana, balm, bay and cinnamon jatoba employing Principal Component Analysis (PCA). The levels of phenolic compounds were centered on the mean and subjected to ACP. The results indicated that the cachaça aged in chestnut (36 months), oak (48 months) cinnamon and bay differed from the others by possessing a higher content of gallic acid, ellagic acid and eugenol, respectively. The samples aged in amburana (48 months), balsam (36 and 48 months), jequitibá, oak (24 and 36 months) and jatoba showed characteristics similar to one another, highlighting the importance of the ACP analysis to show the effect of the type of wood on the phenolic composition of aged cachaça.
\end{abstract}

Additional keywords: cachaça; phenols; wood.

\section{Introdução}

A aguardente de cana é uma bebida fermentodestilada largamente consumida no País. Estima-se que sua produção seja de aproximadamente 1,5 bilhão de litros/ano, sendo o volume exportado representado por apenas $1 \%$ da produção (CARDOSO, 2006). Na busca por novos mercados, produtores têm procurado cada vez mais formas alternativas para agregar valor ao produto, sendo que o envelhecimento tem-se tornado uma prática comum durante o processo de produção da bebida.

Os recipientes utilizados para o envelhecimento são os barris e tonéis de madeira, que atuam como uma membrana semipermeável, permitindo a passagem de vapores de álcool e água, que ocorre em função das condições de umidade relativa e da temperatura do local de armazenamento.

No envelhecimento da bebida, a madeira sofre degradação pela ação do álcool e da água. 
Ocorre hidrólise da hemicelulose e da lignina, sendo que os produtos dessa hidrólise passam para o destilado (SHEREV \& BRINK,1980). Durante o envelhecimento da bebida, a hemicelulose é ligeiramente degradada em pentoses e hexoses, resultando no aumento da concentração de açúcares no produto final (PUECH; MOUTOUNET, 1988).

A lignina tem estreita relação com o desenvolvimento de aroma e sabor nos destilados envelhecidos porque libera para a bebida ácidos e aldeídos aromáticos durante o período de envelhecimento, sendo que pequena fração é solúvel e, consequentemente, extraída durante o processo ${ }^{4}$. Esses compostos podem sofrer reações de oxidação, formando novos compostos aromáticos. Outra reação que pode ocorrer é a esterificação dos álcoois e ácidos, produzindo ésteres, levando as bebidas envelhecidas a apresentarem maiores concentrações destas substâncias (MIRANDA et al., 2006).

Embora o Brasil possua atualmente várias espécies de madeira utilizadas para 0 envelhecimento, como amendoin (Plerogyne nitens), cerejeira (Amburana cearensis), cedro (Cedrela fissilis), jatobá (Hymenaeae carbouril), ipê (Tabebuia sp), freijó (Cordia goeldiana), garapa (Apuleia leiocarpa), bálsamo (Myroxylon peruiferum), vinhático-amarelo (Plathynemia foliosa) e jequitibá (Carinian legalis), os barris de carvalho-europeu (Quercus sp) ainda predominam. No entanto, esta madeira é de difícil aquisição, uma vez que grande parte dos produtores reaproveitam barris que anteriormente foram utilizados para o envelhecimento de uísque, conhaque, vinhos, etc. Outro entrave ao uso dos barris de carvalho é que não se encontram no mercado barris de distintas capacidades (MORI et al., 2003).

Estima-se que o Brasil possua cerca de 30 mil produtores de cachaça, sendo a maioria deles pequenos produtores. Isso implica uma variedade de madeiras que possam estar sendo utilizadas para o envelhecimento de aguardente de cana, exigindo que se estabeleçam estudos para a caracterização química das bebidas provenientes dessas (AQUINO et al., 2006).

Dentre os compostos encontrados na bebida envelhecida, destacam-se os compostos fenólicos. Estes pertencem a uma classe de compostos que possuem, pelo menos, um anel aromático no qual um ou mais hidrogênios são substituídos por grupamentos hidroxila. Dividemse em fenóis simples, ácidos fenólicos, cumarinas, flavonoides, taninos e ligninas (SOARES, 2002).

Os ácidos benzoicos possuem sete átomos de carbono (Ar-COOH) e são os ácidos fenólicos mais simples encontrados na natureza; caracterizam-se por apresentarem um anel benzênico, um grupamento carboxílico e um ou mais grupamentos hidroxila e/ou metóxila na molécula. São pesquisados por conferir propriedades antioxidantes tanto para os alimentos como para o organismo, sendo, por isso, indicados para o tratamento e prevenção do câncer, doenças cardiovasculares e outras doenças. Como exemplo, citam-se os ácidos siríngico, vanílico e o gálico (SOARES, 2002).

Os ácidos cinâmicos, também pertencentes à classe dos ácidos fenólicos, são os precursores da maioria dos compostos classificados como fenilpropanoides $\left(\mathrm{ArC}_{3}\right)$, compostos aromáticos com uma cadeia lateral de três átomos de carbono ligada ao anel aromático. Dentre os ácidos pertencentes a essa classe, destacamse os ácidos cinâmico, o-cumárico, m-cumárico, p-cumárico, cafeico, ferúlico e sinápico, como os ácidos mais encontrados no reino vegetal. Sua cadeia lateral pode sofrer redução, formando compostos como o eugenol ou perder átomos de carbono, originando derivados $\mathrm{ArC}_{2}$ e $\mathrm{ArC}_{1}$ ou sofrer ciclização, dando origem à classe das cumarinas (SIMÕES et al., 2004).

Alguns compostos fenólicos não se apresentam na forma livre nos tecidos vegetais. São encontrados sob a forma de polímeros, na qual se encontram os taninos e as ligninas. Os taninos são compostos fenólicos solúveis em água, de alto peso molecular, que conferem ao alimento a sensação de adstringência, sendo responsável pelo progressivo escurecimento da cor do destilado com o envelhecimento. São classificados em dois grupos, baseados em seu tipo estrutural: taninos hidrolisáveis e taninos condensados (SIMÕES et al., 2004).

A Análise de Componentes Principais é uma ferramenta quimiométrica que permite extrair, de um determinado conjunto de dados, informações relevantes para o seu entendimento. Este conjunto de dados é organizado na forma de matriz, onde as linhas podem ser amostras e as colunas variáveis. Essa técnica permite-nos simplificar e reduzir a dimensão original dos dados, modelar, detectar amostras anômalas (outliers), selecionar variáveis importantes em determinado sistema, classificar e prever comportamentos (MATOS et al., 2003). O que se busca na química de bebidas envelhecidas são marcadores químicos que nos permitam 0 reconhecimento de tal processo e a madeira utilizada.

Com base no exposto, o presente trabaIho objetivou-se comparar a composição fenólica de cachaças envelhecidas em carvalho, jequitibá, castanheira, amburana, bálsamo, lourocanela e jatobá, utilizando-se da Análise das Componentes Principais (ACP). 


\section{Material e métodos}

Obtenção das amostras: as 12 amostras de cachaças utilizadas no experimento foram coletadas em diferentes unidades produtoras da região sul do Estado de Minas Gerais, provenientes de diferentes barris de madeira com capacidades distintas. A Tabela 1 apresenta a origem, tipo de madeira, capacidade do barril e tempo de armazenamento de cada amostra.

Tabela 1 - Locais de coleta e condições de armazenamento das amostras (madeiras, capacidade do barril e tempo de armazenamento). Collecting loci and storage conditions of samples (kind of wood, barrel capacity, and storage time).

\begin{tabular}{cllrc}
\hline Amostra & Local & Madeira & $\begin{array}{r}\text { Capacidade do barril } \\
(\mathrm{L})\end{array}$ & $\begin{array}{c}\text { Tempo de armazenamento } \\
\text { (meses) }\end{array}$ \\
\hline L1 & Lavras & Jequitibá & 10.000 & 48 \\
L2 & Passa Quatro & Castanheira & 700 & 36 \\
L3 & Passa Quatro & Carvalho & 200 & 36 \\
L4 & Passa Quatro & Jequitibá & 20.000 & 36 \\
L5 & Barbacena & Jequitibá & 10.000 & 60 \\
L6 & Lavras & Amburana & 10.000 & 48 \\
L7 & Piranguinho & Bálsamo & 5.000 & 48 \\
L8 & Piranguinho & Louro-canela & 50.000 & 24 \\
L9 & Piranguinho & Carvalho & 50.000 & 48 \\
L10 & Itajubá & Bálsamo & 3.000 & 36 \\
L11 & Itajubá & Jatobá & 4.000 & 6 \\
L12 & Itajubá & Carvalho & 1.000 & 24 \\
\hline
\end{tabular}

\section{Compostos fenólicos}

Reagentes e padrões: os padrões empregados para análise foram: ácido gálico, catequina, ácido vanílico, fenol, ácido siríngico, vanilina, siringaldeído, umbeliferona, ácido p-cumárico, ácido sinápico, ácido m-cumárico, cumarina, 4-metilumbeliferona, ácido o-cumárico, ácido elágico e eugenol, todos adquiridos da Sigma-Aldrich ou da Acros Organics. Todos os compostos químicos utilizados foram de grau analítico para HPLC: metanol (grau HPLC/ Merck), água ultrapura, obtido de um sistema Milli-Q e ácido acético glacial (J.T. Baker) foram usados como solventes para a cromatografia.

Condições cromatográficas e quantificação dos compostos fenólicos: as análises dos compostos fenólicos foram realizadas em um cromatógrafo líquido de alta eficiência Shimadzu, equipado com duas bombas de alta pressão, modelo LC-20AD; um detector com arranjo de diodos (DAD), modelo SPD-M20A; injetor automático com autoamostrador, modelo SIL-M20A; forno modelo CTO-20AC. As separações foram realizadas empregando-se uma coluna empacotada Shim-pack VP-ODS (250 mm x 4,6 mm) com partículas esféricas de $5 \mu \mathrm{m}$ conectada a uma pré-coluna Shim-pack VP-ODS $(5,0 \mathrm{~cm} \times$ 4,0 mm, $5 \mu \mathrm{m}$ - SHIMADZU).

A quantificação dos compostos fenólicos foi realizada utilizando-se do método de padronização externa. O comprimento de onda empregado foi de $280 \mathrm{~nm}$. O fluxo utilizado em toda a análise foi de $1,25 \mathrm{~mL} \mathrm{~min}^{-1}$, e o volume injetado das amostras e do padrão foi de $20 \mu \mathrm{L}$. A eluição foi realizada em sistema do tipo gradiente: 0 a $25 \min (0-40 \%$ B); 25 a 43 min (40-60\%B); 43 a $50 \min (60-100 \%$ B); 50 a $55 \min (100-0 \%$ B); 55 a $60 \mathrm{~min}(0-0 \% \mathrm{~B})$. A fase móvel foi composta por solução de ácido acético a $2 \%$ em água (fase A) e a $70 \%$ metanol e ácido acético a $2 \%$ em água (fase $\mathrm{B}$ ). As amostras foram realizadas em triplicata, e a identidade dos analitos foi confirmada pelo tempo de retenção e pelos picos da amostra, comparados aos dos padrões.

Delineamento estatístico: os dados obtidos foram submetidos à análise de variância, sendo as médias comparadas pelo teste de SchottKnott, ao nível de $95 \%$ de confiança, usando o programa estatístico SISVAR (FERREIRA, 2003).

Análise multivariada: os compostos fenólicos foram quantificados por Cromatografia Líquida de Alta eficiência (CLAE), e os resultados obtidos foram centrados na média e submetidos à Análise das Componentes Principais (ACP). Na aplicação da ACP, foi empregado o MATLAB versão 6.1 (The MathWorks, Natick, USA).

\section{Resultados e discussão}

A Tabela 2 apresenta os teores de compostos fenólicos nas amostras analisadas. De acordo com os valores obtidos para cada composto e comparando-os entre as amostras, podem-se observar diferenças estatísticas significativas. 
De acordo com MIRANDA et al. (2006), diversos fatores podem influenciar na qualidade da aguardente de cana envelhecida, como espécie da madeira, tamanho e pré-tratamento do barril, condições ambientais, tempo de envelhecimento e teor de álcool da bebida.

Quanto às espécies de madeira analisadas, pode-se observar a predominância de diferentes compostos fenólicos. Nas bebidas enveIhecidas em barris de castanheira, temos a predominância dos ácidos gálico $\left(12,15 \mathrm{mg} \mathrm{L}^{-1}\right) \mathrm{e}$ elágico $\left(10,7 \mathrm{mg} \mathrm{L}^{-1}\right) \quad(\mathrm{L} 2)$, na amburanacumarina $\left(11,10 \mathrm{mg} \mathrm{L}^{-1}\right)$, e eugenol $\left(6,65 \mathrm{mg} \mathrm{L}^{-1}\right)$ (L6), no louro-canela catequina $\left(1,26 \mathrm{mg} \mathrm{L}^{-1}\right) \mathrm{e}$ eugenol $\left(2,84 \mathrm{mg} \mathrm{L}^{-1}\right)(\mathrm{L} 8)$, e no jatobá somente foi encontrada a cumarina $\left(0,08 \mathrm{mg} \mathrm{L}^{-1}\right)(\mathrm{L} 11)$. Avaliou-se também a predominância desses compostos em três barris de carvalho, três de jequitibá e dois de bálsamo. Foram encontrados, para os barris de carvalho, teores de siringaldeído $\left(0,95 \mathrm{mg} \mathrm{L}^{-1}\right)$ e ácido elágico $\left(2,08 \mathrm{mg} \mathrm{L}^{-1}\right)$ (L3), ácidos $p$-cumárico $\left(9,19 \mathrm{mg} \mathrm{L}^{-1}\right)$ e elágico (10,5 $\left.\mathrm{mg} \mathrm{L}^{-1}\right)$ (L9), ácidos p-cumárico $\left(1,19 \mathrm{mg} \mathrm{L}^{-1}\right)$ e elágico $\left(1,51 \mathrm{mg} \mathrm{L}^{-1}\right)$ (L12); para os barris de jequitibá, ácidos gálico $\left(1,60 \mathrm{mg} \mathrm{L}^{-1}\right)$ e elágico $\left(1,82 \mathrm{mg} \mathrm{L}^{-1}\right)(\mathrm{L} 1)$, ácidos gálico $\left(1,26 \mathrm{mg} \mathrm{L}^{-1}\right) \mathrm{e}$ elágico $\left(1,60 \mathrm{mg} \mathrm{L}^{-1}\right) \quad(L 4)$, ácido gálico $\left(3,03 \mathrm{mg} \mathrm{L}^{-1}\right)$ e eugenol $\left(3,56 \mathrm{mg} \mathrm{L}^{-1}\right)(\mathrm{L} 5)$, e para os barris de bálsamo, ácido siríngico $\left(3,77 \mathrm{mg} \mathrm{L}^{-1}\right)$ e eugenol $\left(18,42 \mathrm{mg} \mathrm{L}^{-1}\right)$ (L7) e ácidos siríngico $\left(0,40 \mathrm{mg} \mathrm{L}^{-1}\right)$ e elágico $\left(0,41 \mathrm{mg} \mathrm{L}^{-1}\right)(\mathrm{L} 10)$. Pelos dados da Figura 1, observa-se que as cachaças envelhecidas em castanheira (36 meses), carvalho (48 meses) e louro-canela se diferenciaram das demais pelo maior teor de ácido gálico, ácido elágico e eugenol, respectivamente. As amostras envelhecidas em amburana (48 meses), bálsamo (48 meses), jequitibá, carvalho (24 e 36 meses), bálsamo (36 meses) e jatobá apresentaram características semelhantes entre si. Trabalhos similares, como o de Silva et al. (2012), utilizaram-se da quimiometria aliada a resultados espectrofotométricos e cromatográficos para a identificação de extratos hidroalcoólicos de madeiras com base na espécie empregada. Essa técnica também tem sido aplicada para avaliar parâmetros sensoriais durante o processo de envelhecimento da cachaça (CARDELLO; FARIA, 1998) e também para traçar o perfil sensorial por meio dos teores de compostos fenólicos totais, $\mathrm{pH}$, teores de cobre, cor e acidez fixa, volátil, em amostras de cachaças comerciais envelhecidas e sem envelhecer (CARDELLO; FARIA, 2000). Diversos trabalhos utilizam a técnica para a diferenciação de bebidas destiladas em alambiques e em colunas, e a caracterização da bebida por meios dos voláteis nelas presentes (RECHE; FRANCO, 2009; SERAFIM et al., 2011).
As diferenças quanto à presença e à concentração dos compostos fenólicos em cachaças envelhecidas em espécies de madeira diferentes, observadas neste trabalho, corroboram o de Dias et al. (2002). Neste trabalho, eles observaram a predominância de compostos como ácidos elágico e vanílico no carvalho; ácido vanílico e sinapaldeído em amburana; vanilina e ácido elágico no bálsamo; ácido gálico no jequitibá; coniferaldeído no jatobá, e ácidos siríngico e coniferaldeído no ipê. Essas diferenças também podem ser explicadas pelas variáveis como: capacidade do barril e o tempo de armazenamento descritos na Tabela 2. Segundo dados da literatura, o teor de compostos extraídos da madeira aumenta com o tempo de envelhecimento e com - aumento da superfície de contato entre bebida/madeira (MIRANDA et al., 2006; ANJOS et al., 2011). Isto pode ser observado nas amostras L1, L4 e L5, provenientes de barris de jequitibá. No entanto, quando se observam as amostras L3, L9 e L12 (carvalho) e L7 e L10 (bálsamo), este conceito não se aplica. As diferenças observadas entre os teores dos compostos, que são comuns entre as amostras, podem estar relacionadas ao teor alcoólico da bebida. Embora os barris apresentem maior capacidade, diminuindo a superfície de contato, o teor alcoólico da bebida, sendo superior, promove uma extração mais efetiva dos compostos da madeira.

Outro fator relevante é o tempo de uso dos barris. Segundo MORI (2006), a relação é inversamente proporcional entre o teor de compostos fenólicos presente na bebida e o tempo de uso dos barris de madeira no processo de envelhecimento.

Com exceção da amostra envelhecida em barris de jatobá, todas as outras apresentaram certa heterogeneidade quanto à presença dos compostos fenólicos, diferindo apenas quanto ao teor desses. Sabe-se que estes compostos, oriundos da degradação da lignina ou dos extrativos presentes no cerne da madeira, associados aos açúcares provenientes da degradação da hemicelulose e dos produtos provenientes das reações desencadeadas com 0 processo de envelhecimento, são os responsáveis pelo sabor e aroma característicos da bebida envelhecida, diferenciando-a da bebida denominada nova.

A Figura 1 apresenta os gráficos de escores e pesos da ACP obtidos para a composição fenólica das amostras de aguardente. 
Tabela 2 - Concentração $\left(\mathrm{mg} \mathrm{L}^{-1}\right)$ de compostos fenólicos nas amostras de aguardente de cana analisadas. Concentration ( $\mathrm{mg} \mathrm{L}^{-1}$ ) of phenolic compounds in the analyzed sugar cane cachaça samples.

\begin{tabular}{|c|c|c|c|c|c|c|}
\hline $\begin{array}{l}\text { Amostras } \\
\text { Compostos }\end{array}$ & L1 & L2 & L3 & L4 & L5 & L6 \\
\hline Ácido gálico & $\begin{array}{c}1,60 \pm \\
7,12 \times 10^{-4}\end{array}$ & $\begin{array}{c}12,15 \pm \\
2,94 \times 10^{-3} \text { a9 }\end{array}$ & $\begin{array}{c}0,67 \pm \\
3,76 \times 10^{-2} \text { a3 }\end{array}$ & $\begin{array}{c}1,26 \pm \\
7,13 \times 10^{-3}\end{array}$ & $\begin{array}{c}3,03 \pm \\
6,27 \times 10^{-4} \text { a8 }\end{array}$ & $<L Q$ \\
\hline Catequina & $\begin{array}{c}0,39 \pm \\
2,27 \times 10^{-3}\end{array}$ & $\begin{array}{c}1,34 \pm \\
1,44 \times 10^{-2}\end{array}$ & $\begin{array}{c}0,22 \pm \\
2,00 \times 10^{-3}\end{array}$ & $\begin{array}{c}0,28 \pm \\
4,98 \times 10^{-3}\end{array}$ & $\begin{array}{c}0,37 \pm \\
5,59 \times 10^{-3}\end{array}$ & $\begin{array}{c}1,45 \stackrel{ \pm}{ \pm} \\
1,38 \times 10^{-2} \text { a9 }\end{array}$ \\
\hline Ácido vanílico & $\begin{array}{c}0,69 \pm \\
6,07 \times 10^{-3}\end{array}$ & $\begin{array}{c}0,54 \pm \\
9,18 \times 10^{-3} \text { a3 }\end{array}$ & $\begin{array}{c}0,29 \pm \\
1,30 \times 10^{-2} \text { a2 }\end{array}$ & $\begin{array}{c}0,17 \pm \\
3,37 \times 10^{-3} \text { a1 }\end{array}$ & $\begin{array}{c}0,30 \pm \\
7,43 \times 10^{-3} \\
\text { a2 }\end{array}$ & $\begin{array}{c}3,30 \pm \\
7,59 \times 10^{-4} \mathrm{a} 6\end{array}$ \\
\hline Fenol & $\begin{array}{c}0,37 \pm \\
1,38 \times 10^{-2}\end{array}$ & $\begin{array}{c}0,61 \pm \\
4,35 \times 10^{-3}\end{array}$ & $\begin{array}{c}0,71 \pm \\
1,51 \times 10^{-1} \text { a4 }\end{array}$ & $\begin{array}{c}0,34 \pm \\
2,50 \times 10^{-3} \text { a3 }\end{array}$ & $<L Q$ & $\begin{array}{c}0,34 \pm \\
3,75 \times 10^{-3} \text { a3 }\end{array}$ \\
\hline Ácido siríngico & $<\mathrm{LQ}$ & $\begin{array}{c}0,14 \pm \\
1,19 \times 10^{-3} \text { a5 }\end{array}$ & $\begin{array}{c}0,48 \pm \\
6,16 \times 10^{-3} \text { a9 }\end{array}$ & $<L Q$ & $\begin{array}{c}0,16 \pm \\
3,01 \times 10^{-3} \mathrm{a} 6\end{array}$ & $<L Q$ \\
\hline Vanilina & $\begin{array}{c}0,13 \pm \\
1,70 \times 10^{-3} \text { a2 }\end{array}$ & $\begin{array}{c}0,18 \pm \\
2,56 \times 10^{-3} \text { a3 }\end{array}$ & $\begin{array}{c}0,28 \pm \\
1,72 \times 10^{-2}\end{array}$ & $\begin{array}{c}0,11 \pm \\
1,53 \times 10^{-3} \text { a2 }\end{array}$ & $\begin{array}{c}0,20 \pm \\
1,15 \times 10^{-3} \text { a3 }\end{array}$ & $\begin{array}{c}0,24 \pm \\
2,92 \times 10^{-3} \text { a3 }\end{array}$ \\
\hline Siringaldeído & $\begin{array}{c}0,67 \pm \\
7,64 \times 10^{-3} \text { a7 }\end{array}$ & $\begin{array}{c}1,50 \pm \\
1,39 \times 10^{-3} \text { a9 }\end{array}$ & $\begin{array}{c}0,95 \pm \\
1,21 \times 10^{-2} \text { a8 }\end{array}$ & $\begin{array}{c}0,34 \pm \\
2,10 \times 10^{-3} \text { a5 }\end{array}$ & $\begin{array}{c}0,97 \pm \\
3,54 \times 10^{-3} \text { a8 }\end{array}$ & $<L Q$ \\
\hline Umbeliferona & $<L D$ & $<L D$ & ND & ND & $<$ LD & $\begin{array}{c}0,08 \pm \\
1,77 \times 10^{-2}\end{array}$ \\
\hline Ácido p-cumárico & $\begin{array}{c}1,09 \pm \\
6,49 \times 10^{-3}\end{array}$ & $\begin{array}{c}1,30 \pm \\
2,11 \times 10^{-2} \text { a2 }\end{array}$ & ND & $<L Q$ & $\begin{array}{c}1,10 \pm \\
4,23 \times 10^{-3}\end{array}$ & $\begin{array}{c}1,14 \pm \\
1,35 \times 10^{-2}\end{array}$ \\
\hline Ácido sinápico & ND & ND & ND & ND & ND & $\begin{array}{c}1,30 \pm \frac{ \pm}{-3} \\
9,23 \times 10^{-3} \text { a3 }\end{array}$ \\
\hline Ácido m-cumárico & ND & ND & ND & ND & ND & ND \\
\hline Cumarina & $\begin{array}{c}0,77 \pm \\
1,47 \times 10^{-3} \mathrm{a} 6\end{array}$ & $\begin{array}{c}0,11 \pm \\
1,80 \times 10^{-3} \text { a3 }\end{array}$ & $\begin{array}{c}0,14 \pm \\
4,50 \times 10^{-4}\end{array}$ & $\begin{array}{c}0,20 \pm \\
6,56 \times 10^{-4} \text { a5 }\end{array}$ & $\begin{array}{c}0,09 \pm \\
1,06 \times 10^{-3} \mathrm{a} 2\end{array}$ & $\begin{array}{c}11,10 \pm \\
9,26 \times 10^{-4} \text { a7 }\end{array}$ \\
\hline 4metilumbeliferona & $\begin{array}{c}0,51 \pm \\
9,24 \times 10^{-3} \text { a3 }\end{array}$ & $\begin{array}{c}0,38 \pm \\
2,05 \times 10^{-2} \text { a2 }\end{array}$ & ND & ND & ND & $\begin{array}{c}2,32 \pm \\
4,38 \times 10^{-4}\end{array}$ \\
\hline Ácido o-cumárico & $<L Q$ & $<L Q$ & ND & ND & $<L Q$ & $<L Q$ \\
\hline Ácido elágico & $\begin{array}{c}1,82 \pm \\
0,02 \mathrm{a} 6\end{array}$ & $\begin{array}{c}10,70 \pm \\
1,03 \times 10^{-1} \text { a } 9\end{array}$ & $\begin{array}{c}2,08 \pm \\
5,68 \times 10^{-2}\end{array}$ & $\begin{array}{c}1,60 \pm \\
2,64 \times 10^{-2}\end{array}$ & $\begin{array}{c}2,09 \pm \\
5,98 \times 10^{-3}\end{array}$ & $\begin{array}{c}0,83 \pm \\
4,73 \times 10^{-2}\end{array}$ \\
\hline Eugenol & ND & $<L D$ & ND & ND & $\begin{array}{c}3,56 \pm \\
3,81 \times 10^{-1} \\
a 2\end{array}$ & $\begin{array}{c}6,65 \stackrel{ \pm}{ \pm} \\
1,01 \times 10^{-1} \text { a4 } \\
\end{array}$ \\
\hline $\begin{array}{l}\text { Amostras } \\
\text { Compostos } \\
\end{array}$ & L7 & L8 & L9 & L10 & $\mathrm{L} 11$ & $\mathrm{~L} 12$ \\
\hline Ácido gálico & $<L Q$ & ND & $\begin{array}{c}2,78 \pm \\
5,96 \times 10^{-3} \text { a7 }\end{array}$ & ND & ND & $\begin{array}{c}1,03 \pm \\
3,22 \times 10^{-3} \text { a4 }\end{array}$ \\
\hline Catequina & $\begin{array}{c}0,35 \pm \\
7,07 \times 10^{-3} \text { a4 }\end{array}$ & $\begin{array}{c}1,26 \pm \\
5,00 \times 10^{-2} \text { a7 }\end{array}$ & $\begin{array}{c}0,62 \pm \\
1,65 \times 10^{-2} \text { a6 }\end{array}$ & $\begin{array}{c}0,38 \pm \\
1,30 \times 10^{-2} \text { a5 }\end{array}$ & ND & ND \\
\hline Ácido vanílico & $\begin{array}{c}0,60 \pm \\
7,11 \times 10^{-3} \text { a3 }\end{array}$ & $0,68 \pm 3,06 \times 10^{-3}$ a4 & $\begin{array}{c}1,37 \pm \\
2,87 \times 10^{-3} \text { a5 }\end{array}$ & $<L Q$ & $<L D$ & $\begin{array}{c}0,11 \pm \\
4,03 \times 10^{-3}\end{array}$ \\
\hline Fenol & $\begin{array}{c}0,74 \pm \\
4,92 \times 10^{-2}\end{array}$ & $<L Q$ & $\begin{array}{c}1,72 \pm \\
1,26 \times 10^{-2} \text { a6 }\end{array}$ & $\begin{array}{c}0,41 \pm \\
1,09 \times 10^{-2} \text { a3 }\end{array}$ & ND & $\begin{array}{c}0,67 \pm \\
6,58 \times 10^{-4} \text { a4 }\end{array}$ \\
\hline Ácido siríngico & $\begin{array}{c}3,77 \pm \\
2,14 \times 10^{-3} \text { a } 11\end{array}$ & $0,10 \pm 1,55 \times 10^{-2}$ a4 & $\begin{array}{c}1,59 \pm \\
1,51 \times 10^{-3} \text { a } 10\end{array}$ & $\begin{array}{c}0,40 \pm \\
3,73 \times 10^{-3} \text { a8 }\end{array}$ & ND & $\begin{array}{c}0,26 \pm \\
6,12 \times 10^{-3} \text { a7 }\end{array}$ \\
\hline Vanilina & $\begin{array}{c}0,42 \pm \\
3,11 \times 10^{-3} \text { a5 }\end{array}$ & $0,17 \pm 1,75 \times 10^{-3}$ a3 & $\begin{array}{c}1,95 \pm \\
2,66 \times 10^{-3} \mathrm{a} 6\end{array}$ & $\begin{array}{c}0,19 \pm \\
6,86 \times 10^{-3} \text { a3 }\end{array}$ & $<L Q$ & $\begin{array}{c}0,19 \pm \\
1,71 \times 10^{-3} \text { a3 }\end{array}$ \\
\hline Siringaldeído & $<L Q$ & $0,08 \pm 4,53 \times 10^{-3} \mathrm{a} 4$ & $\begin{array}{c}4,65 \pm \\
462 \times 10^{-3} \mathrm{a} 10\end{array}$ & $<L D$ & ND & $\begin{array}{c}0,53 \pm \\
5.32 \times 10^{-4} \mathrm{a}\end{array}$ \\
\hline Umbeliferona & $<L D$ & $0,06 \pm 1,09 \times 10^{-2}$ a3 & ND & ND & ND & ND \\
\hline Ácido p-cumárico & ND & ND & $\begin{array}{c}9,19 \stackrel{ \pm}{-2} \\
5,11 \times 10^{-2} \text { a3 }\end{array}$ & ND & ND & $\begin{array}{c}1,19 \pm \\
3,78 \times 10^{-2} \mathrm{a} 2\end{array}$ \\
\hline Ácido sinápico & $\begin{array}{c}0,51 \pm \\
7,07 \times 10^{-5} \text { a2 }\end{array}$ & ND & $<L D$ & ND & ND & ND \\
\hline Ácido m-cumárico & $\begin{array}{c}1,49 \pm \\
6,91 \times 10^{-2} \text { a3 }\end{array}$ & ND & $\begin{array}{c}0,37 \pm \\
6,84 \times 10^{-4} \text { a2 }\end{array}$ & ND & ND & ND \\
\hline Cumarina & $\begin{array}{c}0,19 \pm \\
1,13 \times 10^{-2} \text { a5 }\end{array}$ & ND & $\begin{array}{c}0,20 \pm \\
1,65 \times 10^{-4} \text { a5 }\end{array}$ & $\begin{array}{c}0,09 \pm \\
4,98 \times 10^{-4} \text { a2 }\end{array}$ & $\begin{array}{c}0,08 \pm \\
1,27 \times 10^{-3} \mathrm{a} 2\end{array}$ & ND \\
\hline 4metilumbeliferona & $<L Q$ & $0,51 \pm 4,77 \times 10^{-2}$ a3 & $<\mathrm{LD}$ & $<L D$ & ND & ND \\
\hline Ácido o-cumárico & $\begin{array}{c}0,14 \pm \\
4,68 \times 10^{-3}\end{array}$ & ND & ND & ND & ND & ND \\
\hline Ácido elágico & $\begin{array}{c}0,64 \pm \\
1,99 \times 10^{-2}\end{array}$ & ND & $\begin{array}{c}10,50 \pm \\
3,18 \times 10^{-1} \text { a8 }\end{array}$ & $\begin{array}{c}0,41 \pm \\
1,15 \times 10^{-3}\end{array}$ & ND & $\begin{array}{c}1,51 \pm \\
6,74 \times 10^{-3} \text { a5 }\end{array}$ \\
\hline Eugenol & $\begin{array}{c}18,42 \pm \\
8,40 \times 10^{-1} \mathrm{a} 5\end{array}$ & $<\mathrm{LQ}$ & $\begin{array}{c}5,76 \pm \\
3,59 \times 10^{-1} \text { a3 } \\
\end{array}$ & ND & ND & ND \\
\hline
\end{tabular}

$<\mathrm{LD}$ = menor que o limite de deteç̧ão; $<\mathrm{LQ}=$ menor que o limite de quantificação; ND = não detectado. 

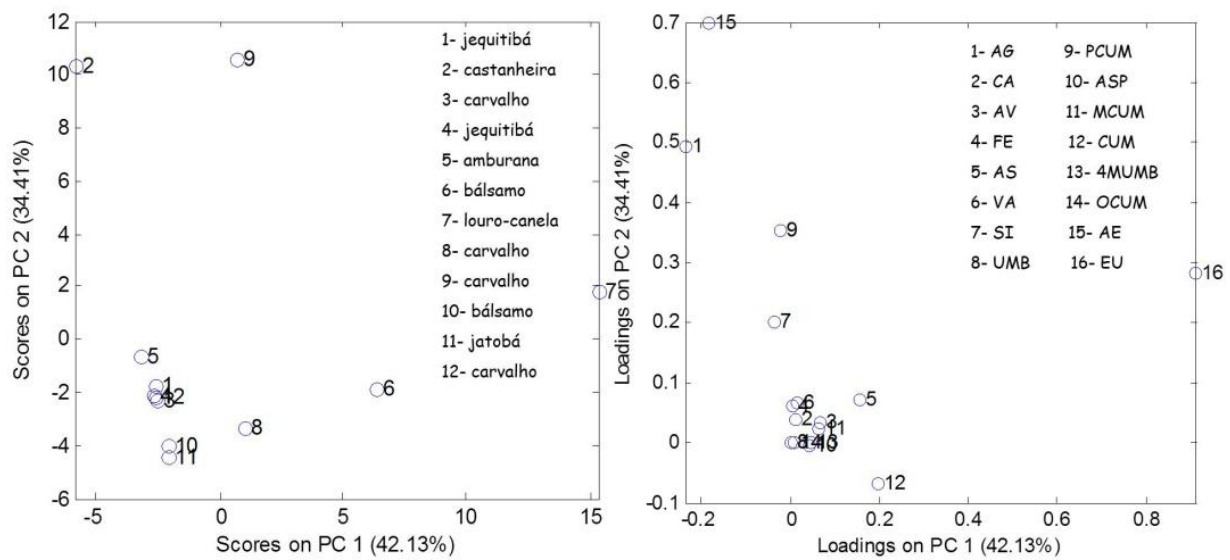

Figura 1 - Análise dos Componentes Principais de aguardentes de cana envelhecida em diferentes barris de madeira. Principal Component Analysis of sugar cane spirits aged in different wooden barrels.

\section{Conclusões}

De acordo com os resultados apresentados, pode-se inferir que a Análise das Componentes Principais (ACP) permitiu evidenciar o efeito da madeira na composição fenólica das cachaças envelhecidas, diferindo daquelas envelhecidas em castanheira (36 meses), carvalho (48 meses) e louro-canela pela presença de ácido gálico, ácido elágico e eugenol, respectivamente, das demais amostras analisadas. Ressalta-se que essa é uma técnica relevante para o conhecimento da química de bebidas envelhecidas juntamente com as técnicas cromatográficas, buscando identificar compostos denominados como marcadores químicos.

\section{Agradecimento}

Os autores agradecem ao Conselho Nacional de Desenvolvimento Científico e Tecnológico ( $\mathrm{CNPq})$, à Fundação de Amparo à Pesquisa do Estado de Minas Gerais (FAPEMIG), aos produtores pelo fornecimento de amostras e ao Departamento de Química da UFLA, pelo suporte nas análises.

\section{Referências}

ANJOS, J. P.; CARDOSO, M. G.; SACZK, A. A.; DOREA, H. S.; SANTIAGO, W. D.; ZACARONI, L. M.; MACHADO, A. M. R.; NELSON, D. L. Evolution of the Concentration of Phenolic Compounds in Cachaça during Aging in an Oak (Quercus sp.) Barrel. Journal of the Brazilian Chemical Society, São Paulo, v.22, p.1307-1314, 2011.
AQUINO, F. W. B.; NASCIMENTO, R. F.; RODRIGUES, S.; CASEMIRO, A. R. S. Determinação de marcadores de envelhecimento em cachaças. Ciência e Tecnologia de Alimentos, Campinas, v.26, n.1, p.145-149, 2006.

CARDELLO, H. M. A. B.; FARIA, J. B. Análise descritiva quantitativa de aguardentes de cana durante o envelhecimento em tonel de carvalho (Quercus alba L.). Revista Ciência e Tecnologia de Alimentos, v.18, n.2, p.169-175, 1998.

CARDELLO, H. M. A. B.; FARIA, J. B. Sensory profile and physicochemical characteristics of commercial brazilian sugar cane spirits, both aged and non-aged. Brazilian Journal of Food Technology, Campinas, n.3, p.31-40, 2000.

CARDOSO, M. G. Produção de aguardente de cana. 2.ed. Lavras: Editora UFLA, 2006.

DIAS, S. M. B. C.; MAIA, A. B. R. A.; NELSON, D. L. Utilização de madeiras nativas no envelhecimento da cachaça de alambique. Informe Agropecuário, Belo Horizonte, v.23, n.217, p.46$51,2002$.

FERREIRA, D. F. Sisvar. Versão 4.6, Build 61. Lavras: DEX/UFLA, 2003.

MATOS, G. D.; PEREIRA-FILHO, E. R.; POPPI, R. J.; ARRUDA, M. A. Z. Análise exploratória em química analítica com emprego de quimiometria: PCA e PCA de imagens. Revista Analytica, São Paulo, n.6, p.38-50, 2003.

MIRANDA, M. B.; HORII, J.; ALCARDE, A. R. Estudo do efeito da irradiação gamma (60Co) na qualidade da cachaça e no tonel de envelhecimento. Ciência e Tecnologia de Alimentos, Campinas, v.26, p.772-778, n.4, 2006. 
MORI, F. A.; MENDES, L. M.; TRUGILHO, P. F.; CARDOSO, M. G. Utilização de eucaliptos e de madeiras nativas no armazenamento de aguardente de cana-de-açúcar. Ciência e Tecnologia de Alimentos, Campinas, v.23, n.3, p.396-400, 2003.

MORI, F. A.; MENDES, L. M.; SILVA, J. R. M.; TRUGILHO, P. F. Influência da qualidade da madeira no envelhecimento da aguardente. In: Produção de aguardente de cana. 2.ed., Lavras: Editora UFLA, 2006. p.243-270.

PUECH, J. L.; MOUTOUNET, M. Liquid chromatographic determination of scopoletin in hydroalcoholic extracts of oak wood and in matured distilled alcoholic beverages. Journal of the Association of Official Analytical Chemists, Arlington, v.71, p.512-514, 1988.

RECHE, R. V.; FRANCO, D. W. Distinção entre cachaças destiladas em alambiques e em colunas usando quimiometria. Quimica Nova, São Paulo, v.32, n.2, p.332-336, 2009.

SERAFIM, F. A. T.; BUCHVISER, S. F.; GALINARO, C. A.; FRANCO, D. W.; NOVAES, F. V. Ácidos Orgânicos em Aguardentes produzidas em alambique e em coluna. Química Nova, São Paulo, v.34, n.1, p.28-32, 2011.
SHEREV, R. N.; BRINK, J. A. Indústria de fermentação. In: SHEREV, R. N. (Ed.). Indústrias de processos químicos. 4.ed., Rio de Janeiro: Guanabara Dois, 1980. cap.31, p.469-495.

SILVA, A. A.; NASCIMENTO, E. S. P.; CARDOSO, D. R.; FRANCO, D. W. Identificação de extratos etanólicos de madeiras utilizando seu espectro eletrônico de absorção e análise multivariada. Química Nova, São Paulo, v.35, n.3, p.563-566, 2012.

SIMÕES, C. M.; SHENKEL, E. P.; GOSMAN, G.; MELLO, J. C. P.; MENTZ, L. A.; PETROVICK, P. R. Farmacognosia: da planta ao medicamento. 5.ed. Porto Alegre: Ed. Da UFRGS, 2004. 1102p.

SINGLETON, V. L. Maturation of wines and spirits: comparison, facts and hypotheses. American Journal of Enology and Viticulture, Davis, v.46, n.1, p.98-115, 1995.

SOARES, S. E. Ácidos fenólicos como antioxidantes. Revista Nutrição, Campinas, v.15, n.1, p.71-81, 2002. 